\title{
SUR LA PRESENCE DU GENRE PROSOPISTOMA AU LIBAN. DESCRIPTION DE P. ORONTI N. SP. ET DE P. PHOENICIUM N. SP. [EPHEMEROPTERA]
}

\author{
par N. J. ALOUF 1
}

\begin{abstract}
Desoription de deux nouvelles espèces de Prosopistoma: P. oronti $\mathbf{n}$. sp. et $P$. phoenicium $\mathrm{n}$. $\mathrm{sp}$. Ces espèces se rencontrent dans deux différents bassins hydrographiques du Liban.
\end{abstract}

\section{On the presence of the Genus Prosopistoma (Ephemeroptera) in Lebanon.}

Description of P. oronti sp. $\mathbf{n}$. and P. phoenicium sp. $\mathbf{n}$.

Two new species of Prosopistoma, $P$. oronti sp. n. und $P$. phoenicium sp. n., are described from two different river systems in Lebanon.

La présence du genre Prosopistoma est signalée en Europe, en Afrique (Lafon 1952, Gillies 1954), en Asie du Sud et dans la région orientale (Peters 1967). Les prospections méthodiques de certains réseaux hydrographiques du Liban, dans le cadre d'une étude subventionnée par le Conseil National de la Recherche Scientifique du Liban sur la faune d'eau douce, permirent la découverte de ce genre dans deux cours d'eau: le 'Assi (Oronte) et le Damour. Malheureusement il n'a pas été possible de capturer la forme adulte et les essais d'élevage se sont soldés par des échecs.

Les adultes de Prosopistoma foliaceum Fourcroy, 1785 et de P. africanum Gillies, 1954 sont seuls connus. Les autres espèces ne sont connues et décrites qu'à l'état larvaire. D'ailleurs, Gillies (1954) avait déjà noté : "It is clear that many characters are available for the separation of the nymphs of the genus, while the ease with which the adults may be separated is still to be discovered. For this reason, the nymph has been designated as the holotype of africanum and no hesitation was felt in describing and naming crassi from nymphal material alone ». Peters (1967) note: "While I agree with Gillies (1954) that many nymphal characters are available to separate the species, most useful characters are extremely minute. Only by careful dissection and use of high power and compound microscopes can most species of Prosopistoma be identified in the nymphs ».

1. Faculté des Sciences de l'Université Libanaise, Hadath-Beyrouth, Liban. 
De ce qui précède, la description des taxa appartenant à ce genre est possible en se basant uniquement sur les caractères larvaires bien qu'il soit évidemment souhaitable de connaître la forme adulte.

\section{1. - Prosopistoma oronti $\mathbf{n}$. sp.}

\section{1. - Matériel eXAMiné}

Nahr-l'Assi (Oronte), proche de la localité Hermel, altitude $600 \mathrm{~m}$. Une larve récoltée le 20 juillet 1974, 38 larves de différentes tailles récoltées entre octobre 1974 et août 1975. Une larve, récoltée le 23-111975 , est considérée comme holotype et déposée, comme les autres larves paratypes, dans ma collection privée à la Faculté des Sciences de l'Université Libanaise.

\section{2. - Description}

La largeur maximale du mésonotum des dix plus grandes larves est de 2,5-3 $\mathrm{mm}$. Il est plus large que long suivant la ligne de suture médiane. La couleur est vert-brun, avec de grandes taches claires (fig. 1). La tête, plus de deux fois plus large que longue, porte trois ocelles; les deux latéraux sont subtriangulaires et aussi grands que les yeux.

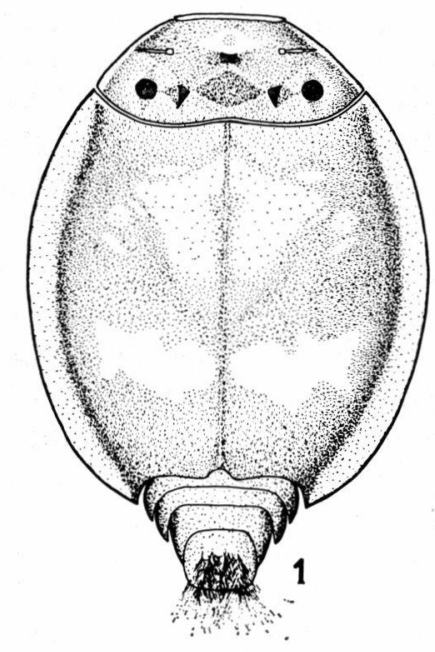

FIG. 1 : Prosopistoma oronti n. sp.

L'antenne est formée de 5, parfois 4 , articles, le second est nettement plus long que l'ensemble des 4 autres (fig. 5). La branche externe de la mandibule a une rangée de $4-5$ dents du côté interne 
et une autre rangée de 6-8 dents du côté externe. La branche interne a deux rangées de 2-3 dents latérales, en plus des deux dents terminales; la base est garnie de 6-8 longues soies serrulées, excepté pour la distale qui est plus courte, plus trapue et plus serrulée que les autres (fig. 2). La maxille est garnie de 4 épines terminales, dont 3 sont très grosses, de 3 soies serrulées et d'une petite soie basale; les 3 articles du palpe sont dans le rapport de longueur de 5, 5 et 2 respectivement (fig. 6). Les 3 articles du palpe labial sont dans le rapport de longueur de 13, 10 et 4 respectivement (fig. 7). Le tibia de la première paire de pattes est tapissé de soies simples sur toute sa surface et est garni de 6-7 fortes soies dentées sur la moitié distale du bord antérieur (fig. 3) ; le fémur est aussi garni de soies (fig. 4).

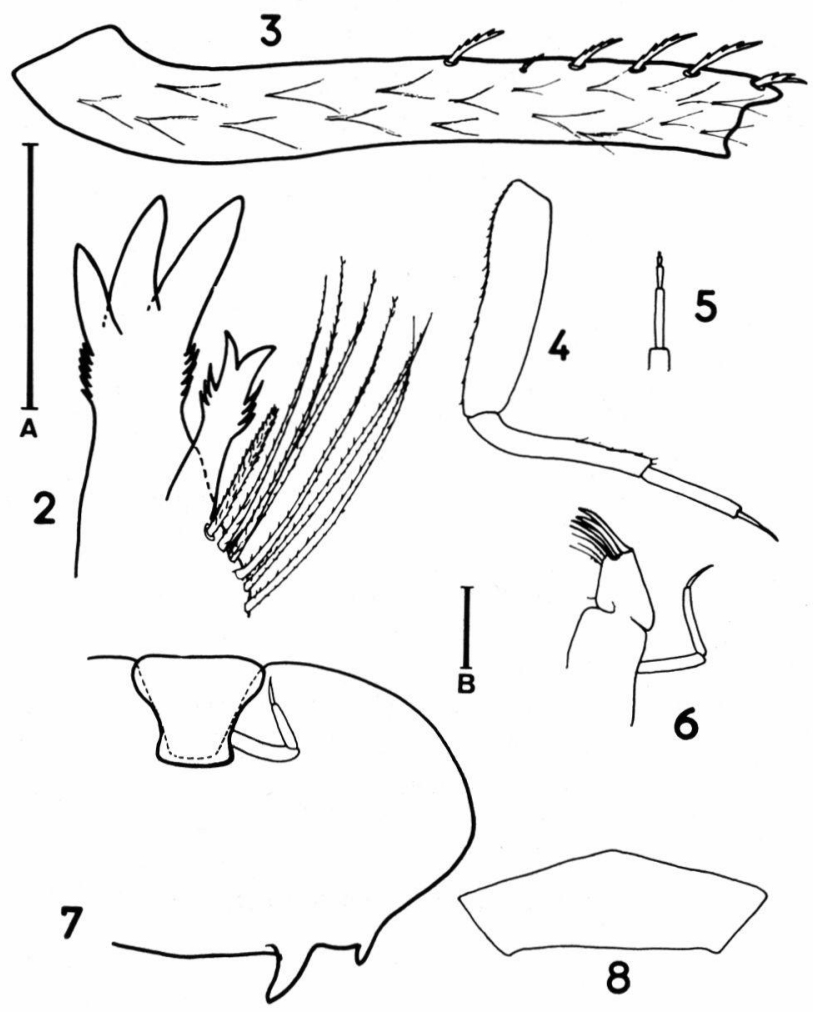

Fig. 2 à 8 : Prosopistoma oronti $n$. sp. -2 : mandibule (échelle A). -3 : tibia de la première patte (échelle $A$ ). -4 : première patte (échelle $B$ ). -5 : antenne (échelle B). - 6: maxille (échelle B). - 7: labium (échelle B). - 8: labre (échelie B). Les traits $\mathrm{A}$ et $\mathrm{B}$ correspondent à $0,2 \mathrm{~mm}$. 
$P$. oronti s'apparente essentiellement à $P$. foliaceum par la forme des ocelles et par la sétation de la mandibule et de la maxille. Il en diffère par le net développement des dents latérales sur les deux branches de la mandibule. Ce dernier caractère permet d'ailleurs de différencier cette espèce de celles de la région orientale, décrites et étudiées par Peters (1967), et de celles de la région éthiopienne (Gillies 1954). P. oronti se distingue aussi de ces dernières par le nombre de soies basales garnissant la mandibule.

\section{3. - BIOLOGIE}

Cette espèce colonise la partie amont à eau rapide de l'Oronte. La largeur du lit oscille autour de $10 \mathrm{~m}$, la profondeur varie entre 25 et $40 \mathrm{~cm}$ à $2 \mathrm{~m}$ du bord. La température varie de 13 à $16^{\circ} \mathrm{C}$. Deux prélèvements sont pris à chaque sortie mensuelle qui a lieu durant la quatrième semaine du mois. Le moyen de récolte est un filet de Surber de $1 / 10 \mathrm{~m}^{2}$ de surface et de 3 mailles par $\mathrm{mm}$. Le fond est raclé avec la main. L'évolution de la taille est représentée sur le graphique I. Un seul prélèvement fut pris en juillet 1974 et les résultats négatifs des mois d'octobre et de novembre seraient dus aux techniques de prospections.

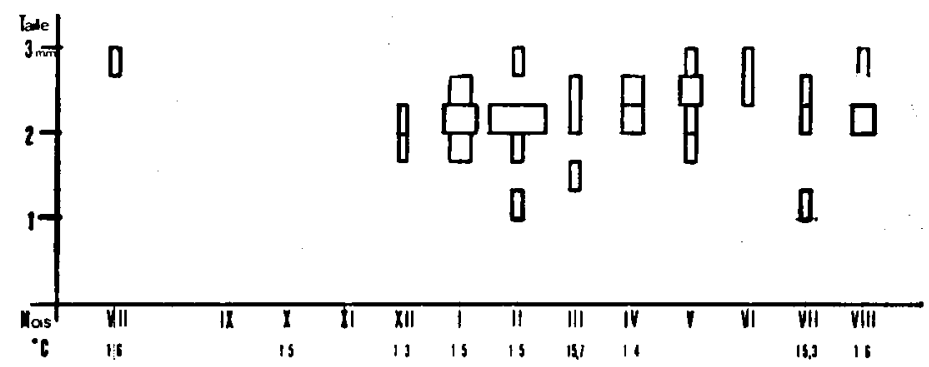

Graphioue I. - Tailles des larves de Prosopistoma oronti capturées aux différents mois.

Bien que le nombre d'individus ne soit pas très important, on peut émettre l'hypothèse que $P$. oronti est une espèce univoltine dont la période de vol s'échelonnerait entre février et août.

\section{2. - Prosopistoma phoenicium n. sp.}

\section{1. - Matériel eXaminé}

Nahr-d-Damour, localité Gisr-1-Cadi, altitude $260 \mathrm{~m}$. Une larve $q$ holotype et une larve paratype récoltées en juin 1973 sont déposées dans ma collection privée à la Faculté des Sciences de l'Université Libanaise. 


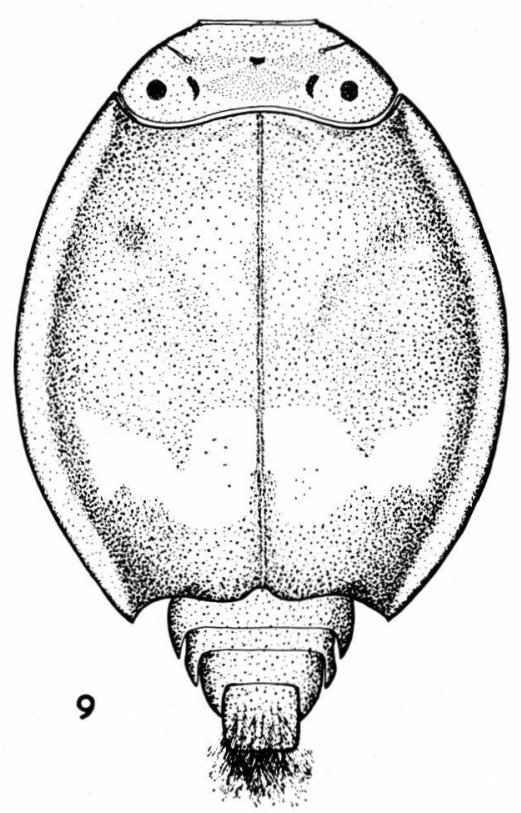

FIG. 9 : Prosopistoma phoenicium n. sp.

\section{2. - DESCRIPTION}

Le mésonotum est de couleur brune et est tacheté de zones claires et sombres. Sa largeur, plus importante que la longueur suivant la ligne de suture médiane, est de $3 \mathrm{~mm}$ pour l'holotype (fig. 9) et de 2,5 mm pour le paratype. La tête est deux fois plus largue que longue. Les ocelles latéraux sont en forme de virgules renversées.

L'antenne est formée de 5 articles qui sont dans le rapport de longueur de 6, 25, 11, 5 et 1 (fig. 14). La branche externe de la mandibule est garnie de deux rangées de 8-9 dents latérales. La branche interne est garnie, en plus des deux dents terminales, de deux dents latérales surmontant des protubérances, la base est garnie de 5 soies serrulées dont la proximale est la plus courte et la distale la plus trapue (fig. 10). La maxille est garnie de 4 épines terminales, 3 grosses et une mince, de 3 soies serrulées et d'une soie basale simple. Les 3 articles du palpe maxillaire sont dans le rapport de longueur de 22, 25 et 11 (fig. 13). Les 3 articles du palpe labial sont dans le rapport de longueur de 12,9 et 4 ( $f$ ig. 15). Le tibia de la première paire de pattes est tapissé de soies sur toute sa surface, le bord antérieur est garni, sur son dernier tiers, de 3 fortes soies dentées et de 2 soies simples (fig. 11). 




Fig. 10 à 15 : Prosopistoma phoenicium n. sp. - 10: mandibule (échelle A.). 11. : tibia de la première patte (échelle A). - 12: première patte (échelle B). - 13: maxille (échelle B). - 14: antenne (échelle B). - 15: labium (échelle B.). Les traits $\mathrm{A}$ et $\mathrm{B}$ correspondent à $0,2 \mathrm{~mm}$.

P. phoenicium diffère des espèces déjà décrites par la double rangée de dents latérales sur la branche externe de la mandibule, elle diffère de $P$. oronti par la sétation de la première paire de pattes, le fémur étant glabre, et par le dessin du mésonotum et de la tête. Ce dernier caractère n'est clair que sur la larve holotype. L'autre larve était probablement sur le point d'effectuer la mue imaginale, une grande tache très sombre a rendu l'examen malaisé. Cette espèce n'a été capturée qu'en deux exemplaires, bien que la station ait été continuellement prospectée entre le mois de juin 1974 et le mois d'août 1975. Le lit de la rivière est large de $4-6 \mathrm{~m}$, la profondeur de l'eau varie entre 10 et $25 \mathrm{~cm}$ en été ; la température varie de $10^{\circ} \mathrm{C}$ en hiver à $20^{\circ} \mathrm{C}$ en été. 
Avec la découverte de ces deux nouveaux taxa, la région paléarctique se trouve colonisée par trois espèces de Prosopistoma, une en Europe et deux au Liban. Si la première de ces espèces est potamophile (Lafon 1952), les deux autres seraient rhitrophiles, selon le sens donné au rhithron par Illies et Botosaneanu (1963).

\section{TRAVAUX CITES}

Gillies (M. T.). 1954. - The adult stages of Prosopistoma Latreille (Ephemeroptera) with descriptions of two new species from Africa. Trans. $R$. ent. Soc. Lond., $105:$ 355-372.

Illies (J.) et Botosaneanu (L.). 1963. - Problèmes et méthodes de la classification et de la zonation écologique des eaux courantes considérées surtout du point de vue faunistique. Mitt. int. Ver. theor. angew. Limnol., 12:1-57.

LaFoN (J.). 1952. - Note sur Prosopistoma foliaceum Fourc. (Ephémoroptère). Bull. Soc. zool. France, 77 (5-6) 425-436.

Peters (W. L.). 1967. - New species of Prosopistoma from the oriental region (Prosopistomaboidea : Ephemeroptera). Tijdschr. Ent., 1110 (9) : 207-222. 